\title{
Beklenen Aralığa Dayanan Aralık Tip II Üssel Bulanık Sayının Aralık Tip II Parametrik Yamuk Bulanık Sayı Yakınsaması
}

\author{
Sinem PEKER ${ }^{1(D)}$ ve Efendi NASİBOV ${ }^{2}$ (D)
}

How to cite: Peker, S., \& Nasibov, E. (2021). Beklenen aralı̆̆a dayanan aralık Tip II üssel bulanık sayının aralık Tip II parametrik yamuk bulanık sayı yakınsaması. Sinop Üniversitesi Fen Bilimleri Dergisi, 6(1), 21-32. https://doi.org/10.33484/sinopfbd.817174

Araștırma Makalesi

Sorumlu Yazar

Sinem PEKER

sinem.peker@yasar.edu.tr

Yazarlara ait ORCID

S.P.: 0000-0003-4700-7595

E.N.: 0000-0002-1889-6410

Received: 27.10 .2020

Accepted: 07.02.2021

\section{$\ddot{\mathbf{O} z}$}

Tip I bulanık sayıları belirsizliği ele almak için bazı karar verme problemlerinde kullanılmaktadır. Tip I bulanık sayılarının üyelik dereceleri adi sayılardır. Ancak gerçek yaşam problemlerinde, üyelik derecelerinin bulanık sayılar ile gösterilebileceği olaylar var olabilir. Bu gibi durumlarda, Tip II bulanık sayıları kullanılabilir. Bulanık sayının daha basit bir formunun kullanılması bazı çalışmalarda karmaşık hesaplamalardan kaçınmak için bir avantaj olarak görülmektedir. $\mathrm{Bu}$ durum dikkate alınarak, bu çalışmada aralık Tip II üssel bulanık sayının aralık Tip II parametrik yamuk bulanık sayı yakınsaması, beklenen aralıkların eşitliklerinin kullanıldığı bir kısıtlı optimizasyon problemi ile bulunmuş ve formüller verilmiştir.

Anahtar Kelimeler: Tip II bulanık sayı, üssel bulanık sayı, parametrik yamuk bulanık sayı, yakınsama, optimizasyon

\section{Interval Type II Parametric Trapezoidal Fuzzy Number Approximation of Interval} Type II Exponential Fuzzy Number Based on The Expected Interval

\footnotetext{
${ }^{1}$ Yaşar Üniversitesi, Fen Edebiyat

Fakültesi, Matematik Bölümü, İzmir, Türkiye

${ }^{2}$ Dokuz Eylül Üniversitesi, Fen Fakültesi, Bilgisayar Bilimleri Bölümü, İzmir, Türkiye
}

$\mathrm{Bu}$ çalışma Creative Commons Attribution 4.0 International License ile lisanslanmıştır

\begin{abstract}
Type I fuzzy numbers are used in some decision-making problems to handle the uncertainty. The membership degrees of Type I fuzzy numbers are crisp numbers. However, the events in which the membership degrees may be shown by fuzzy numbers may exist in the real life problems. In that cases, the Type II fuzzy numbers can be used. The usage of the simpler form of the fuzzy number is seen as an advantage for the avoiding of complex calculations in some studies. Considering this, the interval Type II parametric trapezoidal fuzzy number approximation of the interval Type II exponential fuzzy number has been found by a constrained optimization problem in which the equalities between expected intervals are used and the formulas have been given in this study.
\end{abstract}

Keywords: Type II fuzzy number, exponential fuzzy number, parametric trapezoidal fuzzy number, approximation, optimization

\section{Giriş}

Gerek belirsiz bilginin gerekse tamamlanmamış

bilginin söz konusu olduğu durumlarda bulanık

sayı kullanımına başvurulabilir [1].
Bulanıklığın işlendiği çalışmalarda ise yamuk, üçgen bulanık sayılar sıklıkla kullanılan bulanık sayılar arasındadır. Yamuk bulanık sayı ise parametrik bulanık sayısının özel bir halidir ve 
bulanık sayının sol ve sağ taraf fonksiyonlarının şeklinde bir esneklik sağlar [2].

Literatürde bulanık kümenin yanı sıra bulanık kümenin bir genişlemesi olan sezgisel bulanık kümelerin (intuitionistic fuzzy set) kullanıldığ çalışmalar karar verme problemlerinde ve diğer alanlarda karşımıza çıkmaktadır. Sezgisel bulanık kümeler, üyelik fonksiyonu (membership function) ve üyelik dış1 fonksiyonu (non-membership functions) içerir $[3,4]$. Sezgisel bulanık küme, zamanın dikkate alındığı zamansal sezgisel bulanık kümenin (temporal intuitionistic fuzzy set) bir alt kümesi olarak düşünülebilir [5]. Netrosofik değerler (neutrosophic values), belirsizlik ölçüsünün bağımsız derecesini belirlemede etkin rol oynayabilir [6].

Gerçek yaşam problemlerinde karışık bulanık sayılar karşımıza çıkabilir ve bunların tek bir adi (crisp) sayıya dönüşümü yapılmak istenebilir, ancak bu durum fazla bilgi kaybına neden olabilir. $\mathrm{Bu}$ durumun üstesinden gelebilmek için bulanık sayının daha basit şekildeki haline sahip olan bir bulanık sayıya yakınsaması yapılmak istenebilir [7]. Bunun yanı sira, bir bulanık sayının belli bir bulanık sayıya yakınsamasının yapılması, çalışmalarda işlem kolaylıkları sağlayabilir [8]. Literatürde bu konu ile ilgili çeşitli çalışmalar yapılmıştır [9-14]. Örneğin Ban [15] yaptığ çalışmada verilen Tip I bulanık sayının yamuk bulanık sayısı yakınsamasını oluşturmuştur. Yaptığı çalışmada iki bulanık sayı arasındaki Öklid uzaklığını kullanmış ve iki bulanık sayının beklenen değerlerini birbirine eşit kılan kısıtlı optimizasyon problemi yardımıyla verilen bulanık sayının yakınsaması olan yamuk bulanık sayının parametrelerini elde etmiştir. Ban ve Coroianu [16] bulanık sayıya ait parametreleri dikkate alarak bulanık sayının üçgen bulanık sayı yakınsamasını elde etmişlerdir. Ban ve ark. [1] bulanık sayının ağırlıklı L-R (left-right) yakınsamasını veren bir yöntem sunmuşlardır. Yöntemde Hilbert uzayı ve ortalama Öklid uzaklığı kullanmışlardır. Grzegorzewski ve Winiarska [17] bulanık sayının çekirdek (core) ve taşıyıcısı (support) için ek gereklikleri sağlayan yeni operatörler ile beklenen aralığın korunduğu yamuk bulanık sayı yaklaşımı oluşturmuşlardır. Wang ve Li [18] basit üyelik fonksiyonları kullanarak bulanık sayının yakınsamasını veren yöntem sunmuşlar ve yaklaşım operatörlerinin özelliklerini incelemişlerdir. Zhou ve ark. [19] bir algoritma geliştirmişler ve bulanık kümelerin kisitlı yakınsamalarını oluşturmuşlardır. Ban ve Coroianu [20] bulanık sayının en yakın aralık, üçgen ve yamuk yakınsamalarını oluşturmuşlardır. Yaptıkları çalışmada ortalama öklid uzaklığına (euclidean distance) ve belirsizliğe odaklanmıșlardır [20]. Ban [15] öklid uzaklığını kullanarak, bulanık sayının beklenen aralığını koruyan yakınsama yöntemi ile ilgili çalışma yapmıştır. Nasibov ve Peker [2], bulanık bir sayının parametrik yamuk yakınsamasını öklid uzaklığının kullanıldığı bir yaklaşımla oluşturmuştur. Peker ve Nasibov [21], aralık Tip II genelleştirilmiş çan şekilli bulanık sayısının aralık Tip II parametrik yamuk bulanık sayı yakınsanmasını sunmuşlardır. 
Küme elemanlarının üyelik derecelerinin de bulanık olduğu durumlarda Tip II bulanık sayıları söz konusudur [22]. Klasik (ordinary) bulanık kümeler, Tip II bulanık kümelerin özel halidir ve klasik küme elemanlarının üyelik dereceleri adi (crisp) değerlidir. Belirsizliğin daha çok ele alınması gerektiği durumlarda Tip II bulanık sayı kullanımı daha yararlı olabilir [23]. Bu çalışmada küme elemanlarının üyelik derecelerinin de bulanık olduğu durum dikkate alınarak, iç ve diş fonksiyonlarda beklenen aralık değerlerinin korunduğu aralık Tip II üssel bulanık sayının aralık Tip II parametrik yamuk bulanık sayı yakınsaması oluşturulmuştur. Yakınsamada literatürde bu alanda sık olarak başvurulan uzaklıklardan biri olan öklid uzaklığının bir genişlemesi kullanılarak bir k1sitlı optimizasyon problemi ile aralık Tip II parametrik yamuk bulanık sayı yakınsamasına ait formüller verilmiştir. Aralık Tip II yamuk bulanık sayı yakınsaması, aralık Tip II parametrik yamuk bulanık sayı yakınsamasının özel bir halidir. Oluşturulan yakınsama bazı karar verme problemlerinde karmaşık hesapların yer aldığı işlemlerde hem işlem kolaylığı sağlayabilir hem de üyelik dereceleri de bulanık olduğu için bulanıklığın dikkate alınması açısından bir yarar sunabilir.

\section{Materyal ve Metot}

Parametrik yamuk bulanık sayı aşağıdaki üyelik fonksiyonu ile tanımlanabilir [2],

$$
\mu_{A}(x)=\left\{\begin{array}{ccc}
0 & , & x \leq a_{1}-\sigma \\
1-\left(\frac{a_{1}-x}{\sigma}\right)^{s_{L}} & , & a_{1}-\sigma \leq x \leq a_{1} \\
1 & , & a_{1} \leq x \leq a_{2} \\
1-\left(\frac{x-a_{2}}{\beta}\right)^{s_{R}} & , & a_{2} \leq x \leq a_{2}+\beta \\
0 & , & x \geq a_{2}+\beta
\end{array}\right.
$$

Burada

$L_{A}(\alpha)=a_{1}-(1-\alpha)^{1 / s_{L}} \sigma$

$R_{A}(\alpha)=a_{2}+(1-\alpha)^{1 / s_{R}} \beta$

şeklindedir. Yamuk bulanık sayı parametrik yamuk sayının özel halidir ve yamuk bulanık sayıda $s_{L}=s_{R}=1$ 'dir. (1)'deki üyelik fonksiyonu $a_{1}=a_{2}$ durumunda parametrik üçgen bulanık sayıyı tanımlar.

$A$ bulanık sayının beklenen değeri (4) yardımıyla bulunabilir [16].

$B D(A)=\frac{1}{2}\left(\int_{0}^{1} L_{A}(\alpha) d \alpha+\int_{0}^{1} R_{A}(\alpha) d \alpha\right)$

Buna göre parametrik yamuk bulanık sayının beklenen değeri (5)'te de verildiği gibidir.

$B D\left(A_{\text {yamuk }}\right)=\left(\frac{a_{1}+a_{2}}{2}+\frac{1}{2}\left(\frac{s_{R}}{1+s_{R}} \beta-\frac{s_{L}}{1+s_{L}} \sigma\right)\right)$

Bulanık sayının beklenen aralığı ise

$B A(\mathrm{~A})=\left(\int_{0}^{1} L_{A}(\alpha) d \alpha, \int_{0}^{1} R_{A}(\alpha) d \alpha\right)$

şeklinde yazılabilir [17]. Parametrik yamuk bulanık sayının beklenen aralığı (7)'de tanımlandığı gibidir,

$B A\left(A_{\text {yamuk }}\right)=\left(a_{1}-\frac{s_{L}}{1+s_{L}} \sigma, a_{2}+\frac{s_{R}}{1+s_{R}} \beta\right)$

Gauss (üssel) bulanık sayının üyelik fonksiyonu

$\mu_{B}(x)=\left\{e^{-\frac{1}{2}\left(\frac{b-x}{\delta}\right)^{2}}, \quad-\infty<x<\infty\right.$

olarak tanımlanabilir [24]. Burada $a=L_{A}(1)$ ve $\delta$ ise yayılma değeri ile ilgilidir. 
Sağ ve sol tarafta farklı yayılma değerlerine sahip simetrik olmayan üssel bulanık sayısının üyelik fonksiyonu aşağıdaki gibidir.

$\mu_{B}(x)= \begin{cases}e^{-\frac{1}{2}\left(\frac{b-x}{\delta}\right)^{2}}, & x \leq b \\ e^{-\frac{1}{2}\left(\frac{x-b}{\theta}\right)^{2}}, & , x \geq b\end{cases}$

Sol ve sağ taraf fonksiyonları ise sırasıyla

$L_{B}(\alpha)=b-(-2 \ln \alpha)^{\frac{1}{2}} \delta$

$R_{B}(\alpha)=b+(-2 \ln \alpha)^{\frac{1}{2}} \theta$

şeklindedir.

Tip II bulanık sayısında, $x$ 'in üyelik değerleri de bulanıktır ve aralık Tip II bulanık sayısı iç (lower) ve dış (upper) üyelik fonksiyonları söz konudur [22]. Verilen $x$ değeri için diş fonksiyondan elde edilen üyelik değeri iç fonksiyondan elde edilen üyelik değerinden daha düşük olmayacak şekildedir.

Aralık Tip II parametrik yamuk bulanık sayısı aşağıdaki iç (i indeksi) ve dış (d indeksi) üyelik fonksiyonları ile tanımlanabilir,

$$
\begin{aligned}
& \mu_{\mathrm{A}_{\mathrm{i}}}(\mathrm{x}) \\
& =\left\{\begin{array}{ccc}
0 & \mathrm{x} \leq a_{1_{i}}-\sigma_{\mathrm{i}} \\
1-\left(\frac{a_{1_{i}}-\mathrm{x}}{\sigma_{\mathrm{i}}}\right)^{s_{L_{i}}}, & a_{1_{i}}-\sigma_{\mathrm{i}} \leq \mathrm{x} \leq a_{1_{i}} \\
1 & , & a_{1_{i}} \leq \mathrm{x} \leq a_{2_{i}} \\
1-\left(\frac{x-a_{2_{i}}}{\beta_{\mathrm{i}}}\right)^{s_{R_{i}}}, & , & a_{2_{i}} \leq \mathrm{x} \leq a_{2_{i}}+\beta_{\mathrm{i}} \\
0 & , & \mathrm{x} \geq a_{2_{i}}+\beta_{\mathrm{i}}
\end{array}\right.
\end{aligned}
$$

$$
\begin{aligned}
& \mu_{\mathrm{A}_{d}}(\mathrm{x}) \\
& =\left\{\begin{array}{ccc}
0 & \mathrm{x} \leq a_{1_{d}}-\sigma_{\mathrm{d}} \\
1-\left(\frac{a_{1_{d}}-x}{\sigma_{\mathrm{d}}}\right)^{s_{L_{d}}}, & , & a_{1_{d}}-\sigma_{\mathrm{d}} \leq \mathrm{x} \leq a_{1_{d}} \\
1 & , & a_{1_{d}} \leq \mathrm{x} \leq a_{2_{d}} \\
1-\left(\frac{\mathrm{x}-a_{2_{d}}}{\beta_{d}}\right)^{s_{R_{d}}}, & , & a_{2_{d}} \leq \mathrm{x} \leq a_{2_{d}}+\beta_{d} \\
0 & , & \mathrm{x} \geq a_{2_{d}}+\beta_{d}
\end{array}\right.
\end{aligned}
$$

Aralık Tip II parametrik yamuk bulanık sayısının görsel bir örneği Şekil 1'de verilmiştir.

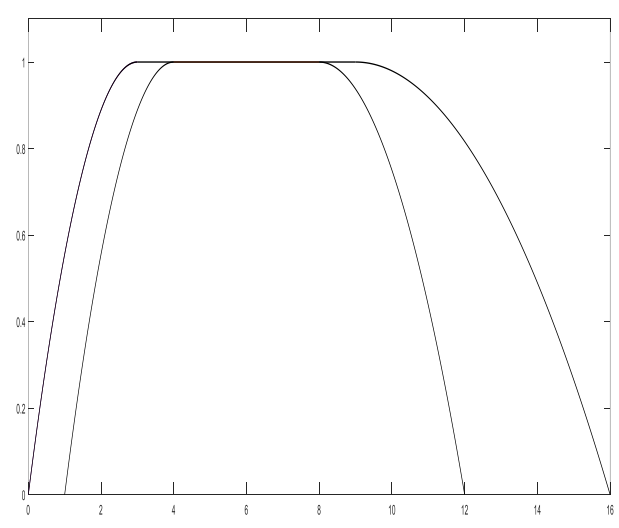

Şekil 1. Aralık Tip II parametrik yamuk bulanık sayısının bir örneği

Aralık Tip II üssel bulanık sayının iç ve dış üyelik fonksiyonları ise (14) ve (15)'de verildiği gibidir.

$\mu_{B_{i}}(x)= \begin{cases}e^{-\frac{1}{2}\left(\frac{b-x}{\delta_{i}}\right)^{2}}, & x \leq b \\ e^{-\frac{1}{2}\left(\frac{x-b}{\theta_{i}}\right)^{2}}, & , x \geq b\end{cases}$

$\mu_{B_{d}}(x)= \begin{cases}e^{-\frac{1}{2}\left(\frac{b-x}{\delta_{d}}\right)^{2}}, & x \leq b \\ e^{-\frac{1}{2}\left(\frac{x-b}{\theta_{d}}\right)^{2}}, & x \geq b\end{cases}$

Aralık Tip II üssel bulanık sayının görsel bir gösterimi Şekil 2'de verilmiştir. 


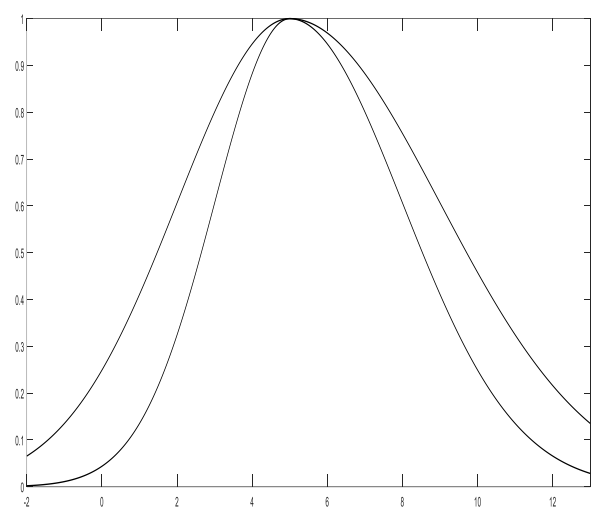

Şekil 2. Aralık Tip II üssel bulanık sayısının bir örneği

Bulanık sayı yakınsama problemlerinde iki bulanık sayı arasındaki uzaklık için genellikle aşağıdaki uzaklık kullanılabilir [17],

$D(A, B)=$

$\sqrt{\int_{0}^{1}\left(\left(L_{\mathrm{A}}(\alpha)-L_{\mathrm{B}}(\alpha)\right)^{2}+\left(R_{\mathrm{A}}(\alpha)-R_{\mathrm{B}}(\alpha)\right)^{2}\right) d \alpha}$.

İki aralık Tip II bulanık sayı arasındaki uzaklık için Öklid uzaklığının bir genişlemesi olan aşağıdaki tanım kullanılabilir.

$D(A, B)=$

$\sqrt{\int_{0}^{1}\left(\begin{array}{c}\left(L_{A_{i}}(\alpha)-L_{B_{i}}(\alpha)\right)^{2}+\left(L_{A_{d}}(\alpha)-L_{B_{d}}(\alpha)\right)^{2} \\ +\left(R_{A_{i}}(\alpha)-R_{B_{i}}(\alpha)\right)^{2}+\left(R_{A_{d}}(\alpha)-R_{B_{d}}(\alpha)\right)^{2}\end{array}\right) d \alpha}$

\section{Bulgular}

Aralık Tip II Simetrik Olmayan Üssel

Bulanık Sayısının Aralık Tip II

Parametrik Yamuk Bulanı Sayı

Yakınsaması

Simetrik olmayan gauss bulanık sayının üyelik fonksiyonunun altında kalan alan sol ve sağ taraf için sırasıyla $\int_{-\infty}^{b} e^{-\frac{1}{2}\left(\frac{b-x}{\delta}\right)^{2}} d x=\frac{\delta \sqrt{\pi}}{\sqrt{2}}$

$\int_{b}^{\infty} e^{-\frac{1}{2}\left(\frac{x-b}{\theta}\right)^{2}} d x=\frac{\theta \sqrt{\pi}}{\sqrt{2}}$

şeklindedir. Bu çalışmada, simetrik olmayan üssel bulanık sayının beklenen aralığını bulmak için herhangi verilen bir bulanık sayı için $\int_{0}^{1} L_{A}(\alpha) d \alpha, \int_{0}^{1} R_{A}(\alpha) d \alpha \quad$ integrallerine karşılık gelebilecek aşağıdaki eşitlikler dikkate alınmıştır.

$\int_{0}^{1} L_{A}(\alpha) d \alpha=L_{A}(1)-\int_{\mathrm{L}_{A}(0)}^{\mathrm{L}_{A}(1)} \underline{\mu}_{A}(x) d x$

$\int_{0}^{1} R_{A}(\alpha) d \alpha=R_{A}(1)+\int_{\mathrm{R}_{A}(1)}^{\mathrm{R}_{A}(0)} \bar{\mu}_{A}(x) d x$

Burada $\underline{\mu}_{A}(x)$ ve $\bar{\mu}_{A}(x)$ sirasiyla $L_{A}(\alpha)$ ve $R_{A}(\alpha)$ 'nın ters fonksiyonlarıdır. $B$ simetrik olmayan üssel bulanık sayı için (20)-(21) eşitliklerinde, $\mathrm{L}_{B}(0)$ ve $\mathrm{R}_{B}(0)$ yerine sırasıyla $-\infty$ ve $\infty$ kullanıldığında ilgili eşitlikler simetrik olmayan üssel bulanık sayı için

$\int_{0}^{1} L_{B}(\alpha) d \alpha=L_{B}(1)-\frac{\delta \sqrt{\pi}}{\sqrt{2}}$

$\int_{0}^{1} R_{B}(\alpha) d \alpha=R_{B}(1)+\frac{\theta \sqrt{\pi}}{\sqrt{2}}$

şeklinde yazılmıştır. Buna göre simetrik olmayan üssel bulanık sayının beklenen aralığı

$B A(\mathrm{~B})=\left(b-\frac{\delta \sqrt{\pi}}{\sqrt{2}}, \mathrm{~b}+\frac{\theta \sqrt{\pi}}{\sqrt{2}}\right)$

olarak alınmıştır.

$\mathrm{Bu}$ çalışmada verilen aralık Tip II simetrik olmayan üssel bulanık sayının aralık Tip II parametrik yamuk bulanık sayı yakınsaması dikkate alınmış, iç ve dış fonksiyonlarına bağlı olan beklenen değerlerini birbirine eşitleyen ve iki bulanık sayı arasındaki $\alpha$-kesitlerine bağlı uzaklığı en küçük yapan üyelik fonksiyon 
değerleri elde edilmeye çalışılmıştır. Üssel fonksiyonun yapisindan kaynaklanan problemlerin üstesinden gelebilmek için (17)'deki uzaklık yerine aşağıdaki amaç fonksiyonu kullanılmıştır.

$F=\sum_{j=1}^{n}\left(\left(L_{A_{i}}\left(\alpha_{j}\right)-L_{B_{i}}\left(\alpha_{j}\right)\right)^{2}+\left(L_{A_{d}}\left(\alpha_{j}\right)-\right.\right.$ $\left.L_{B_{d}}\left(\alpha_{j}\right)\right)^{2}+\left(R_{A_{i}}\left(\alpha_{j}\right)-R_{B_{i}}\left(\alpha_{j}\right)\right)^{2}+\left(R_{A_{d}}\left(\alpha_{j}\right)-\right.$ $\left.\left.R_{B_{d}}\left(\alpha_{j}\right)\right)^{2}\right)$

Burada

$L_{A_{i}}\left(\alpha_{j}\right)=a_{1_{i}}-\left(1-\alpha_{j}\right)^{\frac{1}{s_{L_{i}}}} \sigma_{i}$

$R_{A_{i}}\left(\alpha_{j}\right)=a_{2 i}+\left(1-\alpha_{j}\right)^{\frac{1}{S_{R_{i}}}} \beta_{i}$

$L_{A_{d}}\left(\alpha_{j}\right)=a_{1_{d}}-\left(1-\alpha_{j}\right)^{\frac{1}{s_{L_{d}}}} \sigma_{\mathrm{d}}$

$R_{A_{d}}\left(\alpha_{j}\right)=a_{2_{d}}+\left(1-\alpha_{j}\right)^{\frac{1}{s_{R_{d}}}} \beta_{d}$

$L_{B_{i}}\left(\alpha_{j}\right)=b-\left(-2 \ln \alpha_{j}\right)^{\frac{1}{2}} \delta_{i}$

$R_{B_{i}}\left(\alpha_{j}\right)=b+\left(-2 \ln \alpha_{j}\right)^{\frac{1}{2}} \theta_{i}$

$L_{B_{d}}\left(\alpha_{j}\right)=b-\left(-2 \ln \alpha_{j}\right)^{\frac{1}{2}} \delta_{d}$

$R_{B_{d}}\left(\alpha_{j}\right)=b+\left(-2 \ln \alpha_{j}\right)^{\frac{1}{2}} \theta_{d}$

şeklindedir.

(25)'de yer alan amaç fonksiyonuna en küçük değerini veren ve ilgili iki bulanık sayının beklenen değerlerini birbirine eşit kılan optimizasyon problemi aşağıdaki gibi oluşturulmuştur.

$$
\begin{aligned}
& \min \left(\sum _ { j = 1 } ^ { n } \left(\left(L_{A_{i}}\left(\alpha_{j}\right)-L_{B_{i}}\left(\alpha_{j}\right)\right)^{2}+\left(L_{A_{d}}\left(\alpha_{j}\right)-\right.\right.\right. \\
& \left.L_{B_{d}}\left(\alpha_{j}\right)\right)^{2}+\left(R_{A_{i}}\left(\alpha_{j}\right)-R_{B_{i}}\left(\alpha_{j}\right)\right)^{2}+\left(R_{A_{d}}\left(\alpha_{j}\right)-\right. \\
& \left.\left.\left.R_{B_{d}}\left(\alpha_{j}\right)\right)^{2}\right)\right) \\
& \text { klsitlar: } a_{1_{i}}-\frac{s_{L_{i}}}{1+s_{L_{i}}} \sigma_{i}=b-\frac{\delta_{i} \sqrt{\pi}}{\sqrt{2}} \\
& a_{2_{i}}+\frac{s_{R_{i}}}{1+s_{R_{i}}} \beta_{i}=b+\frac{\theta_{i} \sqrt{\pi}}{\sqrt{2}}
\end{aligned}
$$

$a_{1_{d}}-\frac{s_{L_{d}}}{1+s_{L_{d}}} \sigma_{d}=\mathrm{b}-\frac{\delta_{d} \sqrt{\pi}}{\sqrt{2}}$

$a_{2 d}+\frac{s_{R_{d}}}{1+s_{R_{d}}} \beta_{d}=b+\frac{\theta_{d} \sqrt{\pi}}{\sqrt{2}}$

(35)-(38)'deki kısıtları sağlayacak şekilde (34) de yer alan amaç fonksiyonunun en küçük $a_{1_{i}}$, $a_{2_{i}}, \quad a_{1_{d}}, a_{2_{d}}, \sigma_{i}, \quad \beta_{i}, \sigma_{\mathrm{d}}, \quad \beta_{d}$ parametre değerlerini bulmak için $\lambda_{1}, \lambda_{2}, \lambda_{3}, \lambda_{4}$ lagranj çarpanları olmak üzere aşağıdaki $L$ amaç fonksiyonunun en küçük değerini veren parametre değerleri elde edilmeye çalışılmıştır.

$$
\begin{aligned}
& \mathrm{L}=\mathrm{F}-\lambda_{1}\left(a_{1_{i}}-\frac{s_{L_{i}}}{1+s_{L_{i}}} \sigma_{i}-b+\frac{\delta_{i} \sqrt{\pi}}{\sqrt{2}}\right)-\lambda_{2}\left(a_{2_{i}}+\right. \\
& \left.\frac{s_{R_{i}}}{1+s_{R_{i}}} \beta_{i}-b-\frac{\theta_{i} \sqrt{\pi}}{\sqrt{2}}\right) \\
& \quad-\lambda_{3}\left(a_{1_{d}}-\frac{s_{L_{d}}}{1+s_{L_{d}}} \sigma_{d}-b+\frac{\delta_{d} \sqrt{\pi}}{\sqrt{2}}\right)- \\
& \lambda_{4}\left(a_{2_{d}}+\frac{s_{R_{d}}}{1+s_{R_{d}}} \beta_{d}-b-\frac{\theta_{d} \sqrt{\pi}}{\sqrt{2}}\right)
\end{aligned}
$$

Bunun için $\frac{\partial L}{\partial a_{1_{i}}}=0, \frac{\partial L}{\partial \lambda_{1}}=0, \frac{\partial L}{\partial \sigma_{i}}=0, \frac{\partial L}{\partial a_{2_{i}}}=$ $0, \frac{\partial L}{\partial \lambda_{2}}=0, \frac{\partial L}{\partial \beta_{i}}=0, \frac{\partial L}{\partial a_{1_{d}}}=0, \frac{\partial L}{\partial \lambda_{3}}=0, \frac{\partial L}{\partial \sigma_{d}}=$ $0, \frac{\partial L}{\partial a_{2}}=0, \frac{\partial L}{\partial \lambda_{4}}=0, \frac{\partial L}{\partial \beta_{d}}=0 \quad$ eşitlikleri çözülmüş ve bilinmeyen parametreleri veren eşitlikler elde edilmiştir.

Teorem İç ve dış fonksiyonların beklenen değerlerini (35)-(38)'de verildiği gibi birbirine eşitleyen ve (34)'de verilen amaç fonksiyonunun en küçük değerini veren, aralık Tip II simetrik olmayan üssel bulanık sayının aralık Tip II parametrik yamuk bulanık sayı yakınsamasının $a_{1_{i}}, a_{2_{i}}, a_{1_{d}}, a_{2_{d}}, \sigma_{i}, \beta_{i}, \sigma_{\mathrm{d}}, \beta_{d}$ bilinmeyen parametre değerlerini veren eşitlikler aşağıdaki gibidir:

$a_{1_{i}}=\frac{s_{L_{i}}}{1+s_{L_{i}}} \sigma_{i}+\mathrm{b}-\frac{\delta_{i} \sqrt{\pi}}{\sqrt{2}}$ 


$$
\begin{aligned}
& a_{2_{i}}=-\frac{s_{R_{i}}}{1+s_{R_{i}}} \beta_{i}+b+\frac{\theta_{i} \sqrt{\pi}}{\sqrt{2}} \\
& \sigma_{i}= \\
& \frac{\frac{\left(1+s_{L_{i}}\right)}{{ }_{L_{i}}}\left(\left(-\frac{\delta_{i} \sqrt{\pi}}{\sqrt{2}}\right) \sum_{j=1}^{n}\left(1-\alpha_{j}\right)^{\frac{1}{s_{L_{i}}}}+\delta_{i} \sum_{i=1}^{n}\left(-2 \ln \alpha_{j}\right)^{\frac{1}{2}}\left(1-\alpha_{j}\right)^{\frac{1}{s_{L_{i}}}}\right)}{\left(\left(\frac{s_{L_{i}}}{1+s_{L_{i}}}\right) \frac{n(n+1)}{2}-2 \sum_{j=1}^{n}\left(1-\alpha_{j}\right)^{\frac{1}{s_{L_{i}}}}+\left(\frac{1+s_{L_{i}}}{s_{L_{i}}}\right)\left(\sum_{j=1}^{n}\left(1-\alpha_{j}\right)^{\frac{2}{s_{L_{i}}}}\right)\right)}+ \\
& \frac{-\left(-\frac{\delta_{i} \sqrt{\pi}}{\sqrt{2}}\right) \frac{n(n+1)}{2}-\delta_{i} \sum_{j=1}^{n}\left(-2 \ln \alpha_{j}\right)^{\frac{1}{2}}}{\left(\left(\frac{s_{L_{i}}}{1+s_{L_{i}}}\right) \frac{n(n+1)}{2}-2 \sum_{j=1}^{n}\left(1-\alpha_{j}\right)^{\frac{1}{s_{L_{i}}}}+\left(\frac{1+s_{L_{i}}}{s_{L_{i}}}\right)\left(\sum_{j=1}^{n}\left(1-\alpha_{j}\right)^{\frac{2}{s_{L_{i}}}}\right)\right)}
\end{aligned}
$$

$$
\beta_{i}=
$$

$$
\begin{gathered}
\frac{-\left(\frac{1+s_{R_{i}}}{s_{R_{i}}}\right)\left(\left(\frac{\theta_{i} \sqrt{\pi}}{\sqrt{2}}\right) \sum_{j=1}^{n}\left(1-\alpha_{j}\right)^{\frac{1}{s_{R_{i}}}}-\theta_{i} \sum_{j=1}^{n}\left(-2 \ln \alpha_{j}\right)^{\frac{1}{2}}\left(1-\alpha_{j}\right)^{\frac{1}{s_{R_{i}}}}\right)}{\left(\frac{s_{R_{i}} n(n+1)}{1+s_{R_{i}}}-2 \sum_{j=1}^{n}\left(1-\alpha_{j}\right)^{\frac{1}{s_{R_{i}}}}+\frac{1+s_{R_{i}}}{s_{R_{i}}} \sum_{j=1}^{n}\left(1-\alpha_{j}\right)^{\frac{2}{s_{R_{i}}}}\right)} \\
+\frac{\left(\left(\frac{\theta_{i} \sqrt{\pi}}{\sqrt{2}}\right) \frac{n(n+1)}{2}-\theta_{i} \sum_{j=1}^{n}\left(-2 \ln \alpha_{j}\right)^{\frac{1}{2}}\right)}{\left(\frac{s_{R_{i}} n(n+1)}{1+s_{R_{i}}}-2 \sum_{j=1}^{n}\left(1-\alpha_{j}\right)^{\frac{1}{s_{R_{i}}}}+\frac{1+s_{R_{i}}}{s_{R_{i}}} \sum_{j=1}^{n}\left(1-\alpha_{j}\right)^{\frac{2}{s_{R_{i}}}}\right)}
\end{gathered}
$$

$$
\begin{aligned}
& a_{1_{d}}=\frac{s_{L_{d}}}{1+s_{L_{d}}} \sigma_{d}+\mathrm{b}-\frac{\delta_{d} \sqrt{\pi}}{\sqrt{2}} \\
& a_{2_{d}}=-\frac{s_{R_{d}}}{1+s_{R_{d}}} \beta_{d}+b+\frac{\theta_{d} \sqrt{\pi}}{\sqrt{2}}
\end{aligned}
$$

$\sigma_{d}=$

$\frac{\frac{\left(1+s_{L_{d}}\right.}{s_{L_{d}}}\left(\left(-\frac{\delta_{d} \sqrt{\pi}}{\sqrt{2}}\right) \sum_{j=1}^{n}\left(1-\alpha_{j}\right)^{\frac{1}{s_{L_{d}}}}+\delta_{d} \sum_{i=1}^{n}\left(-2 \ln \alpha_{j}\right)^{\frac{1}{2}\left(1-\alpha_{j}\right)^{\frac{1}{s_{L}}}}\right)}{\left(\left(\frac{s_{L_{d}}}{1+s_{L_{d}}}\right) \frac{n(n+1)}{2}-2 \sum_{j=1}^{n}\left(1-\alpha_{j}\right)^{\frac{1}{s_{L_{d}}}}+\left(\frac{1+s_{L_{d}}}{s_{L_{d}}}\right)\left(\sum_{j=1}^{n}\left(1-\alpha_{j}\right)^{\frac{2}{s_{L_{d}}}}\right)\right)}+$

$$
\frac{-\left(-\frac{\delta_{d} \sqrt{\pi}}{\sqrt{2}}\right) \frac{n(n+1)}{2}-\delta_{d} \sum_{j=1}^{n}\left(-2 \ln \alpha_{j}\right)^{\frac{1}{2}}}{\left(\left(\frac{s_{L_{d}}}{1+s_{L_{d}}}\right) \frac{n(n+1)}{2}-2 \sum_{j=1}^{n}\left(1-\alpha_{j}\right)^{\frac{1}{s_{L_{d}}}}+\left(\frac{1+s_{L_{d}}}{s_{L_{d}}}\right)\left(\sum_{j=1}^{n}\left(1-\alpha_{j}\right)^{\frac{2}{s_{L_{d}}}}\right)\right)}
$$

$$
\lambda_{1}=\frac{2\left(1+s_{L_{i}}\right) \sum_{i=1}^{n}\left(a_{1_{i}}-\left(1-\alpha_{j}\right)^{\frac{1}{S_{L_{i}}}} \sigma_{i}-b+\left(-2 \ln \alpha_{j}\right)^{\frac{1}{2}} \delta_{i}\right)\left(1-\alpha_{j}\right)^{\frac{1}{S_{L_{i}}}}}{s_{L_{i}}}
$$

(48) deki eşitlikte $a_{1_{i}}$ ve $\lambda$ yerine konulduğunda $\beta_{d}=$

$$
\begin{gathered}
\frac{-\left(\frac{1+s_{R_{d}}}{s_{R_{d}}}\right)\left(\left(\frac{\theta_{d} \sqrt{\pi}}{\sqrt{2}}\right) \sum_{j=1}^{n}\left(1-\alpha_{j}\right)^{\frac{1}{s_{R}}}-\theta_{d} \sum_{j=1}^{n}\left(-2 \ln \alpha_{j}\right)^{\frac{1}{2}}\left(1-\alpha_{j}\right)^{\frac{1}{s_{R}}}\right)}{\left(\frac{s_{R_{d}} n(n+1)}{1+s_{R_{d}}}-2 \sum_{j=1}^{n}\left(1-\alpha_{j}\right)^{\frac{1}{s_{R}}}+\frac{1+s_{R_{d}}}{s_{R_{d}}} \sum_{j=1}^{n}\left(1-\alpha_{j}\right)^{\frac{2}{s_{R}}}\right)}+ \\
+\left(\left(\frac{\theta_{d} \sqrt{\pi}}{\sqrt{2}}\right) \frac{n(n+1)}{2}-\theta_{d} \sum_{j=1}^{n}\left(-2 \ln \alpha_{j}\right)^{\frac{1}{2}}\right) \\
\frac{\left.{\frac{s}{R_{d}} n(n+1)}_{1+s_{R_{d}}}^{2}-2 \sum_{j=1}^{n}\left(1-\alpha_{j}\right)^{\frac{1}{s_{R}}}+\frac{1+s_{R_{d}}}{s_{R_{d}}} \sum_{j=1}^{n}\left(1-\alpha_{j}\right)^{\frac{2}{s_{R}}}\right)}{}
\end{gathered}
$$

Burada, $s_{L_{i}}, s_{R_{i}}, s_{L_{d}}, s_{R_{d}}$ seçimlerinde her alfa seviyesi için $L_{A_{d}}\left(\alpha_{j}\right) \leq L_{A_{i}}\left(\alpha_{j}\right) \leq R_{A_{i}}\left(\alpha_{j}\right) \leq$ $R_{A_{d}}\left(\alpha_{j}\right)$ ve $L_{B_{d}}\left(\alpha_{j}\right) \leq L_{B_{i}}\left(\alpha_{j}\right) \leq R_{B_{i}}\left(\alpha_{j}\right) \leq$ $R_{B_{d}}\left(\alpha_{j}\right)$ koşulları sağlanmalıdır.

İspat (39) nolu eşitlik dikkate alındığında $\frac{\partial L}{\partial a_{1_{i}}}=0, \frac{\partial L}{\partial \sigma_{i}}=0, \frac{\partial L}{\partial \lambda_{1}}=0$ eşitlikleri sıra ile çözüldüğü zaman

$$
\begin{aligned}
& 2 \sum_{i=1}^{n}\left(a_{1_{i}}-\left(1-\alpha_{j}\right)^{\frac{1}{s_{L_{i}}}} \sigma_{i}-b+\left(-2 \ln \alpha_{j}\right)^{\frac{1}{2}} \delta_{i}\right)- \\
& \lambda_{1}=0 \\
& -2 \sum_{i=1}^{n}\left(a_{1_{i}}-\left(1-\alpha_{j}\right)^{\frac{1}{s_{L_{i}}}} \sigma_{i}-b+\right. \\
& \left.\left(-2 \ln \alpha_{j}\right)^{\frac{1}{2}} \delta_{i}\right)\left(1-\alpha_{j}\right)^{\frac{1}{S_{L_{i}}}}+\lambda_{1} \frac{s_{L_{i}}}{1+s_{L_{i}}}=0 \\
& a_{1_{i}}-\frac{s_{L_{i}}}{1+s_{L_{i}}} \sigma-b+\frac{\delta_{i} \sqrt{\pi}}{\sqrt{2}}=0
\end{aligned}
$$

eşitlikleri elde edilir. (50) eşitliğinden aşağıdaki $a_{1_{i}}$ değerine ulaş1ır.

$a_{1_{i}}=\frac{s_{L_{i}}}{1+S_{L_{i}}} \sigma_{i}+b-\frac{\delta_{i} \sqrt{\pi}}{\sqrt{2}}$

(49) eşitliğinden ise $\lambda_{1}$ çıkartılabilir. 


$$
\begin{aligned}
\sum_{i=1}^{n}\left(\frac{s_{L_{i}}}{1+s_{L_{i}}} \sigma_{i}+b-\right. & \left.\frac{\delta_{i} \sqrt{\pi}}{\sqrt{2}}-\left(1-\alpha_{j}\right)^{\frac{1}{s_{L_{i}}}} \sigma_{i}-b+\left(-2 \ln \alpha_{j}\right)^{\frac{1}{2}} \delta_{i}\right) \\
- & \frac{\left(1+s_{L_{i}}\right) \sum_{i=1}^{n}\left(\frac{s_{L_{i}}}{1+s_{L_{i}}} \sigma_{i}+\mathrm{b}-\frac{\delta_{i} \sqrt{\pi}}{\sqrt{2}}-\left(1-\alpha_{j}\right)^{\frac{1}{s_{L_{i}}}} \sigma_{i}-b+\left(-2 \ln \alpha_{j}\right)^{\frac{1}{2}} \delta_{i}\right)\left(1-\alpha_{j}\right)^{\frac{1}{s_{L_{i}}}}}{s_{L_{i}}}=0
\end{aligned}
$$

elde edilebilir. Buradan

$$
\begin{aligned}
\sigma_{i}\left(\sum _ { j = 1 } ^ { n } \left(\frac{s_{L_{i}}}{1+s_{L_{i}}}\right.\right. & \left.\left.-\left(1-\alpha_{j}\right)^{\frac{1}{s_{L_{i}}}}\right)+\left(-\frac{1+s_{L_{i}}}{s_{L_{i}}}\right)\left(\sum_{i=1}^{n}\left(\frac{s_{L_{i}}}{1+s_{L_{i}}}\left(1-\alpha_{j}\right)^{\frac{1}{s_{L_{i}}}}-\left(1-\alpha_{j}\right)^{\frac{2}{s_{L_{i}}}}\right)\right)\right) \\
& +\sum_{i=1}^{n}\left(-\frac{\delta_{i} \sqrt{\pi}}{\sqrt{2}}+\left(-2 \ln \alpha_{j}\right)^{\frac{1}{2}} \delta_{i}\right)-\frac{\left(1+s_{L_{i}}\right)}{s_{L_{i}}}\left(\sum_{i=1}^{n}\left(-\frac{\delta_{i} \sqrt{\pi}}{\sqrt{2}}+\left(-2 \ln \alpha_{j}\right)^{\frac{1}{2}} \delta_{i}\right)\left(1-\alpha_{j}\right)^{\frac{1}{s_{L_{i}}}}\right)=0
\end{aligned}
$$

ve

$$
\begin{gathered}
\sigma_{i}\left(\left(\frac{s_{L_{i}}}{1+s_{L_{i}}}\right) \frac{n(n+1)}{2}-2 \sum_{j=1}^{n}\left(1-\alpha_{j}\right)^{\frac{1}{s_{L_{i}}}}+\left(\frac{1+s_{L_{i}}}{s_{L_{i}}}\right)\left(\sum_{j=1}^{n}\left(1-\alpha_{j}\right)^{\frac{2}{s_{i}}}\right)\right)+\left(-\frac{\delta_{i} \sqrt{\pi}}{\sqrt{2}}\right) \frac{n(n+1)}{2}+\delta_{i} \sum_{j=1}^{n}\left(-2 \ln \alpha_{j}\right)^{\frac{1}{2}} \\
-\frac{\left(1+s_{L_{i}}\right)}{s_{L_{i}}}\left(\left(-\frac{\delta_{i} \sqrt{\pi}}{\sqrt{2}}\right) \sum_{j=1}^{n}\left(1-\alpha_{j}\right)^{\frac{1}{s_{L_{i}}}}+\delta_{i} \sum_{i=1}^{n}\left(-2 \ln \alpha_{j}\right)^{\frac{1}{2}}\left(1-\alpha_{j}\right)^{\frac{1}{S_{L_{i}}}}\right)=0
\end{gathered}
$$

sonucuna ulaşılır ve aşağıdaki eşitlik bulunur.

$$
\sigma_{i}=\frac{\left(\begin{array}{c}
\frac{\left(1+s_{L_{i}}\right.}{s_{L_{i}}}\left(\left(-\frac{\delta_{i} \sqrt{\pi}}{\sqrt{2}}\right) \sum_{j=1}^{n}\left(1-\alpha_{j}\right)^{\frac{1}{S_{L_{i}}}}+\delta_{i} \sum_{i=1}^{n}\left(-2 \ln \alpha_{j}\right)^{\frac{1}{2}}\left(1-\alpha_{j}\right)^{\frac{1}{S_{L_{i}}}}\right) \\
-\left(-\frac{\delta_{i} \sqrt{\pi}}{\sqrt{2}}\right) \frac{n(n+1)}{2}-\delta_{i} \sum_{j=1}^{n}\left(-2 \ln \alpha_{j}\right)^{\frac{1}{2}}
\end{array}\right)}{\left(\left(\frac{s_{L_{i}}}{1+s_{L_{i}}}\right) \frac{n(n+1)}{2}-2 \sum_{j=1}^{n}\left(1-\alpha_{j}\right)^{\frac{1}{S_{L_{i}}}}+\left(\frac{1+s_{L_{i}}}{S_{L_{i}}}\right)\left(\sum_{j=1}^{n}\left(1-\alpha_{j}\right)^{\frac{2}{S_{L_{i}}}}\right)\right)}
$$

Benzer şekilde yine (39)'da yer alan eşitlik ve $\frac{\partial L}{\partial a_{2_{i}}}=0, \frac{\partial L}{\partial \lambda_{2}}=0, \frac{\partial L}{\partial \beta_{i}}=0$ eşitlikleri dikkate

$$
\begin{gathered}
2 \sum_{j=1}^{n}\left(a_{2 i}+\left(1-\alpha_{j}\right)^{\frac{1}{s_{R_{i}}}} \beta_{i}-b-\left(-2 \ln \alpha_{j}\right)^{\frac{1}{2}} \theta_{i}\right)(1 \\
\left.-\alpha_{j}\right)^{\frac{1}{s_{R_{i}}}}-\lambda_{2} \frac{s_{R_{i}}}{1+s_{R_{i}}}=0
\end{gathered}
$$
alındığında sırasıyla

$$
\begin{gathered}
2 \sum_{j=1}^{n}\left(a_{2_{i}}+\left(1-\alpha_{j}\right)^{\frac{1}{s_{i}}} \beta_{i}-b-\left(-2 \ln \alpha_{j}\right)^{\frac{1}{2}} \theta_{i}\right) \\
-\lambda_{2}=0 \\
a_{2_{i}}+\frac{s_{R_{i}}}{1+s_{R_{i}}} \beta_{i}-b-\frac{\theta_{i} \sqrt{\pi}}{\sqrt{2}}=0
\end{gathered}
$$

elde edilir. Yukarıdaki ilk iki eşitlikten $\lambda_{2}$

$$
\begin{aligned}
& =2 \sum_{j=1}^{n}\left(a_{2_{i}}+\left(1-\alpha_{j}\right)^{\frac{1}{s_{R_{i}}}} \beta_{i}-b-\left(-2 \ln \alpha_{j}\right)^{\frac{1}{2}} \theta_{i}\right) \\
& a_{2_{i}}=-\frac{s_{R_{i}}}{1+s_{R_{i}}} \beta_{i}+R_{B_{i}}(1)+\frac{\theta_{i} \sqrt{\pi}}{\sqrt{2}} .
\end{aligned}
$$

Bunları üçüncü denklemde yerine koyduğumuzda 


$$
\begin{aligned}
2 \sum_{j=1}^{n}\left(-\frac{s_{R_{i}}}{1+s_{R_{i}}} \beta_{i}+b+\frac{\theta_{i} \sqrt{\pi}}{\sqrt{2}}+\left(1-\alpha_{j}\right)^{\frac{1}{S_{R_{i}}}} \beta_{i}\right. \\
\left.-b-\left(-2 \ln \alpha_{j}\right)^{\frac{1}{2}} \theta_{i}\right)\left(1-\alpha_{j}\right)^{\frac{1}{s_{R_{i}}}} \\
-2 \sum_{j=1}^{n}\left(-\frac{s_{R_{i}}}{1+s_{R_{i}}} \beta_{i}+\mathrm{b}+\frac{\theta_{i} \sqrt{\pi}}{\sqrt{2}}\right. \\
+\left(1-\alpha_{j}\right)^{\frac{1}{s_{R_{i}}}} \beta_{i}-b \\
\left.-\left(-2 \ln \alpha_{j}\right)^{\frac{1}{2}} \theta_{i}\right) \frac{s_{R_{i}}}{1+s_{R_{i}}}=0
\end{aligned}
$$

ve buradan da

$\beta_{i}\left(\left(\frac{s_{R_{i}}}{1+s_{R_{i}}}\right)^{2} \frac{n(n+1)}{2}-\frac{s_{R_{i}}}{1+s_{R_{i}}} \sum_{j=1}^{n}\left(1-\alpha_{j}\right)^{\frac{1}{s_{i}}}-\right.$

$\left.\frac{s_{R_{i}}}{1+s_{R_{i}}} \sum_{j=1}^{n}\left(1-\alpha_{j}\right)^{\frac{1}{s_{R_{i}}}}+\sum_{j=1}^{n}\left(1-\alpha_{j}\right)^{\frac{2}{s_{R_{i}}}}\right)+$

$\left(\left(\frac{\theta_{i} \sqrt{\pi}}{\sqrt{2}}\right) \sum_{j=1}^{n}\left(1-\alpha_{j}\right)^{\frac{1}{s_{R_{i}}}}-\theta_{i} \sum_{j=1}^{n}\left(-2 \ln \alpha_{j}\right)^{\frac{1}{2}}(1-\right.$

$\left.\alpha_{j}\right)^{\frac{1}{s_{i}}}-\left(\frac{s_{R_{i}}}{1+s_{R_{i}}}\right)\left(\left(\frac{\theta_{i} \sqrt{\pi}}{\sqrt{2}}\right) \frac{n(n+1)}{2}-\right.$

$\left.\left.\theta_{i} \sum_{j=1}^{n}\left(-2 \ln \alpha_{j}\right)^{\frac{1}{2}}\right)\right)=0$

elde edilir. Aşağıdaki $\beta_{i}$ değeri bu eşitlikten çıkartılabilir.

$\beta_{i}=$

$$
\frac{\left(\begin{array}{c}
\left.-\left(\frac{1+s_{R_{i}}}{s_{R_{i}}}\right)\left(\left(\frac{\theta_{i} \sqrt{\pi}}{\sqrt{2}}\right) \sum_{j=1}^{n}\left(1-\alpha_{j}\right)^{\frac{1}{s_{R_{i}}}}-\theta_{i} \sum_{j=1}^{n}\left(-2 \ln \alpha_{j}\right)^{\frac{1}{2}\left(1-\alpha_{j}\right.}\right)^{\frac{1}{s_{R_{i}}}}\right) \\
+\left(\left(\frac{\theta_{i} \sqrt{\pi}}{\sqrt{2}}\right) \frac{n(n+1)}{2}-\theta_{i} \sum_{j=1}^{n}\left(-2 \ln \alpha_{j}\right)^{\frac{1}{2}}\right)
\end{array}\right)}{\left(\frac{s_{R_{i}} n(n+1)}{1+s_{R_{i}}}-2 \sum_{j=1}^{n}\left(1-\alpha_{j}\right)^{\frac{1}{S_{R_{i}}}}+\frac{1+s_{R_{i}}}{s_{R_{i}}} \sum_{j=1}^{n}\left(1-\alpha_{j}\right)^{\frac{2}{s_{R_{i}}}}\right)} .
$$

Benzer şekilde, $a_{1_{d}}, a_{2_{d}}, \sigma_{\mathrm{d}}, \beta_{d}$ ispatlarıda yapılabilir.

Örnek Aşağıdaki iç ve dış üyelik fonksiyonları ile tanımlanan aralık Tip II üssel bulanık sayısı ele alınsın.
$\mu_{B_{i}}(x)= \begin{cases}e^{-\frac{1}{2}\left(\frac{3-x}{2}\right)^{2}}, & x \leq 3 \\ e^{-\frac{1}{2}\left(\frac{x-3}{4}\right)^{2}}, & x \geq 3\end{cases}$

$\mu_{B_{d}}(x)= \begin{cases}e^{-\frac{1}{2}\left(\frac{3-x}{3}\right)^{2}}, & x \leq 3 \\ e^{-\frac{1}{2}\left(\frac{x-3}{5}\right)^{2}} & , \quad x \geq 3\end{cases}$

(14) ve (15)'de yer alan aralık Tip II üssel bulanık sayının iç ve dış üyelik fonksiyonları dikkate alındığında,

$b=3, \delta_{i}=2, \theta_{i}=4, \delta_{d}=3, \theta_{d}=5$

olduğu görülmektedir.

Yakınsamada $s_{L_{i}}=1.7, s_{R_{i}}=2, s_{L_{d}}=1.5$, $s_{R_{d}}=2$ olarak alınsın ve $\alpha_{1}=0.1, \alpha_{2}=0.2$, $\alpha_{3}=0.3, \alpha_{4}=0.4, \alpha_{5}=0.5, \alpha_{6}=0.6, \alpha_{7}=$ 0.7, $\alpha_{8}=0.8, \alpha_{9}=0.9, \alpha_{10}=1$ kesitlerinde çalışılsın.

Buna göre (42), (43), (40), (41) nolu eşitliklerinde $b, \delta_{i}, \theta_{i}, s_{L_{i}}, s_{R_{i}}$ değerleri yerine konulduğunda sırasıyla

$\sigma_{i}=$

$$
\frac{\frac{(1+1.7)}{1.7}\left(\left(-\frac{2 \sqrt{\pi}}{\sqrt{2}}\right) \sum_{j=1}^{10}\left(1-\alpha_{j}\right)^{\frac{1}{1.7}}+2 \sum_{i=1}^{n}\left(-2 \ln \alpha_{j}\right)^{\frac{1}{2}}\left(1-\alpha_{j}\right)^{\frac{1}{1.7}}\right)}{\left(\left(\frac{1.7}{1+1.7}\right) \frac{10(10+1)}{2}-2 \sum_{j=1}^{10}\left(1-\alpha_{j}\right)^{\frac{1}{1.7}}+\left(\frac{1+1.7}{1.7}\right)\left(\sum_{j=1}^{10}\left(1-\alpha_{j}\right)^{\frac{2}{1.7}}\right)\right)}+
$$$$
\frac{-\left(-\frac{2 \sqrt{\pi}}{\sqrt{2}}\right)^{\frac{10(10+1)}{2}}-2 \sum_{j=1}^{10}\left(-2 \ln \alpha_{j}\right)^{\frac{1}{2}}}{\left(\left(\frac{1.7}{1+1.7}\right)^{10(10+1)} \frac{1}{2}-2 \sum_{j=1}^{10}\left(1-\alpha_{j}\right)^{\frac{1}{1.7}}+\left(\frac{1+1.7}{1.7}\right)\left(\sum_{j=1}^{10}\left(1-\alpha_{j}\right)^{\frac{2}{1.7}}\right)\right)}
$$

$\beta_{i}=\frac{-\left(\frac{1+2}{2}\right)\left(\left(\frac{4 \sqrt{\pi}}{\sqrt{2}}\right) \sum_{j=1}^{10}\left(1-\alpha_{j}\right)^{\frac{1}{2}}-4 \sum_{j=1}^{10}\left(-2 \ln \alpha_{j}\right)^{\frac{1}{2}}\left(1-\alpha_{j}\right)^{\frac{1}{2}}\right)}{\left(\frac{210(10+1)}{1+2}-2 \sum_{j=1}^{10}\left(1-\alpha_{j}\right)^{\frac{1}{2}}+\frac{1+2}{2} \sum_{j=1}^{10}\left(1-\alpha_{j}\right)^{\frac{2}{2}}\right)}$

$+\frac{\left(\left(\frac{4 \sqrt{\pi}}{\sqrt{2}}\right) \frac{10(10+1)}{2}-4 \sum_{j=1}^{10}\left(-2 \ln \alpha_{j}\right)^{\frac{1}{2}}\right)}{\left(\frac{210(10+1)}{1+2}-2 \sum_{j=1}^{10}\left(1-\alpha_{j}\right)^{\frac{1}{2}}+\frac{1+2}{2} \sum_{j=1}^{10}\left(1-\alpha_{j}\right)^{\frac{2}{2}}\right)}$

$a_{1_{i}}=\frac{1.7}{1+1.7} \sigma_{i}+3-\frac{2 \sqrt{\pi}}{\sqrt{2}}$ 
$a_{2_{i}}=-\frac{2}{1+2} \beta_{i}+3+\frac{4 \sqrt{\pi}}{\sqrt{2}}$

elde edilir.

Benzer şekilde (46), (47), (44), (45) numaralı

eşitliklerinde $b, \delta_{d}, \theta_{d}, s_{L_{d}}, s_{R_{d}}$ değerleri yerine

konularak hesaplamalar yapılır.

$$
\begin{aligned}
& \mu_{\mathrm{A}_{\mathrm{i}}}(\mathrm{x})=\left\{\begin{array}{ccc}
0 & \mathrm{x} \leq-0.98662 \\
1-\left(\frac{3.009358-\mathrm{x}}{3.995978}\right)^{1.7} & , & -0.98662 \leq \mathrm{x} \leq 3.009358 \\
1 & , & 3.009358 \leq \mathrm{x} \leq 3.035028 \\
1-\left(\frac{x-3.035028}{7.465437}\right)^{2} & , & 3.035028 \leq \mathrm{x} \leq 10.50046 \\
0 & , & \mathrm{x} \geq 10.50046
\end{array}\right. \\
& \mu_{\mathrm{A}_{d}}(\mathrm{x})=\left\{\begin{array}{ccc}
0 & \mathrm{x} \leq-3.26964 \\
1-\left(\frac{3.006989-x}{6.276631}\right)^{1.5} & , & -3.26964 \leq \mathrm{x} \leq 3.006989 \\
1 & , & 3.006989 \leq \mathrm{x} \leq 3.110975 \\
1-\left(\frac{\mathrm{x}-3.110975}{9.23101}\right)^{2} & , & 3.110975 \leq \mathrm{x} \leq 12.34199 \\
0 & , & \mathrm{x} \geq 12.34199
\end{array}\right.
\end{aligned}
$$

Elde edilen aralık Tip II parametrik yamuk bulanık sayı Şekil 3'te gösterilmiştir

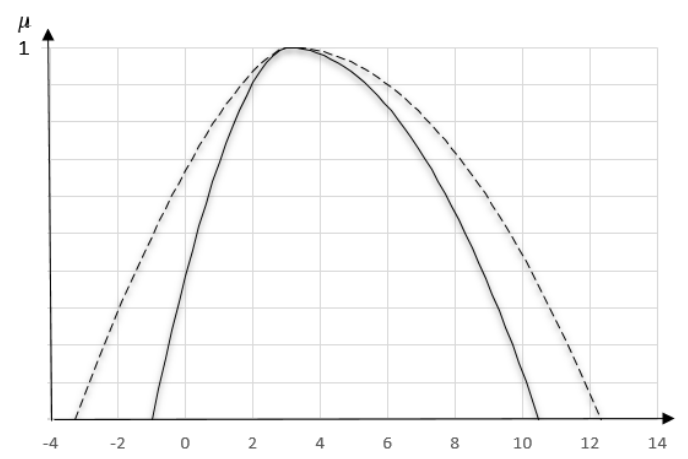

Şekil 3. Aralık Tip II parametrik yamuk bulanık sayı

\section{Tartıșma ve Sonuç}

Belirsizlik, çalışmalarda sıklıkla ortaya çıkabilecek bir kavramdır. Bulanık sayı ise bu belirsizliğin ele alınmasında avantajlar sağlar. Bununla birlikte, karmaşık bulanık sayılar çalışmalarda bazı zorluklar çıkartabilir ve bu durumun üstesinden gelebilmek için bu
İlgili hesaplamalar sonunda, aralık Tip II parametrik yamuk bulanık sayının iç ve dış üyelik fonksiyonları aşağıdaki gibi bulunur.

sayıların daha standart formları olan yakınsamaları tercih edilebilir. Bazı çalışmalarda, belirsizlik daha çok ele alınmak istenebilir ve üyelik derecelerinin de bulanık olduğu Tip II bulanık sayıları süreçlerde kullanılabilir. Bu durumları dikkate alınarak, bu çalışmada aralık Tip II üssel bulanık sayının aralık Tip II parametrik yamuk bulanık sayı yakınsaması kısıtlı optimizasyon problemi ile elde edilmeye çalışmış ve bilinmeyen parametre değerlerini veren formüller bulunmuştur. Optimizasyon probleminde beklenen aralıkların eşitliği kısıt olarak alınmıştır. Önerilen yöntem bir örnek üzerinde gösterilmiştir.

\section{Teşekkür -}

Fon/Finansman bilgileri Yazarlar bu çalışmanın araştırması, yazarlığı veya 
yayınlanması için herhangi bir mali destek almamışlardır.

\section{Etik Kurul Onayı ve İzinler -}

Çıkar çatışmaları/Çatışan çıkarlar Yazarlar çıkar çatışması olmadığını beyan eder.
Yazarların Katkısı Tüm yazarlar, bu çalışmanın planlanmasına, yürütülmesine veya analizine yazar olarak dahil edilmek üzere yeterince katkıda bulunmuştur. Tüm yazarlar makalenin son halini okumuş ve onaylamıştır.

\section{Kaynaklar}

[1] Ban, A. I., Coroianu, L., \& Khastan, A. (2016). Conditioned weighted L-R approximations of fuzzy numbers. Fuzzy Sets and Systems, 283, 56-82. https://doi.org/10.1016/j.fss.2015.03.012

[2] Nasibov, E. N., \& Peker, S. (2008). On the nearest parametric approximation of a fuzzy number. Fuzzy Sets and Systems, 159(11), 1365-1375. https://doi.org/10.1016/j.fss.2007.08.005

[3] Hao, Z., Xu, Z., Zhao, H., \& Zhang, R. (2021). The context-based distance measure for intuitionistic fuzzy set with application in marine energy transportation route decision making. Applied Soft Computing, 101, Article 107044. https://doi.org/10.1016/j.asoc.2020.107044

[4] Krawczak, M., \& Szkatuła, G. (2020). On matching of intuitionistic fuzzy sets. Information Sciences, 517, 254-274. https://doi.org/10.1016/j.ins.2019.11.050

[5] Alcantud, J. C. R., Khameneh, A. Z., \& Kilicman, A. (2020). Aggregation of infinite chains of intuitionistic fuzzy sets and their application to choices with temporal intuitionistic fuzzy information. Information Sciences, 514, 106-117. https://doi.org/10.1016/j.ins.2019.12.008

[6] Sodenkamp, M. A., Tavana, M., \& Di Caprio, D. (2018). An aggregation method for solving group multi-criteria decision-making problems with single-valued neutrosophic sets. Applied Soft Computing, 71, 715-727. https://doi.org/10.1016/j.asoc.2018.07.020

[7] Grzegorzewski, P. (2002). Nearest interval approximation of a fuzzy number. Fuzzy Sets and systems, 130(3), 321-330. https://doi.org/10.1016/S0165-0114(02)00098-2

[8] Hai, S., Gong, Z., \& Chen, Z. (2020). Weighted pseudometric approximation of 2-dimensional fuzzy numbers by fuzzy 2-cell prismoid numbers preserving the centroid. Fuzzy Sets and Systems, 387, 158173. https://doi.org/10.1016/j.fss.2018.12.013

[9] Liu, X., \& Lin, H. (2007). Parameterized approximation of fuzzy number with minimum variance weighting functions. Mathematical and computer modelling, 46(11-12), 1398-1409. https://doi.org/10.1016/j.mcm.2007.01.011

[10] Coroianu, L., \& Stefanini, L. (2016). General approximation of fuzzy numbers by F-transform. Fuzzy Sets and Systems, 288, 46-74. https://doi.org/10.1016/j.fss.2015.03.015

[11] Chanas, S. (2001). On the interval approximation of a fuzzy number. Fuzzy Sets and Systems, 122(2), 353-356. https://doi.org/10.1016/S0165-0114(00)00080-4

[12] Coroianu, L., Gagolewski, M., \& Grzegorzewski, P. (2013). Nearest piecewise linear approximation of fuzzy numbers. Fuzzy Sets and Systems, 233, 26-51. https://doi.org/10.1016/j.fss.2013.02.005

[13] Yeh, C. T., \& Chu, H. M. (2014). Approximations by LR-type fuzzy numbers. Fuzzy Sets and Systems, 257, 23-40. https://doi.org/10.1016/j.fss.2013.09.004 
[14] Huang, H., Wu, C., Xie, J., \& Zhang, D. (2017). Approximation of fuzzy numbers using the convolution method. Fuzzy Sets and Systems, 310, 14-46. https://doi.org/10.1016/j.fss.2016.06.010

[15] Ban, A. (2008). Approximation of fuzzy numbers by trapezoidal fuzzy numbers preserving the

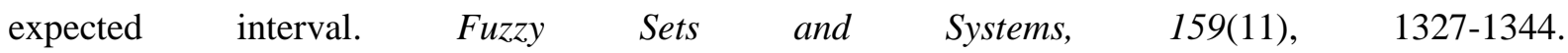
https://doi.org/10.1016/j.fss.2007.09.008

[16] Ban, A. I., \& Coroianu, L. (2015). Existence, uniqueness, calculus and properties of triangular approximations of fuzzy numbers under a general condition. International Journal of Approximate Reasoning, 62, 1-26. https://doi.org/10.1016/j.ijar.2015.05.004

[17] Grzegorzewski, P., \& Pasternak-Winiarska, K. (2014). Natural trapezoidal approximations of fuzzy numbers. Fuzzy Sets and Systems, 250, 90-109. https://doi.org/10.1016/j.fss.2014.03.003

[18] Wang, G., \& Li, J. (2017). Approximations of fuzzy numbers by step type fuzzy numbers. Fuzzy sets and systems, 310, 47-59. https://doi.org/10.1016/j.fss.2016.08.003

[19] Zhou, J., Miao, D., Gao, C., Lai, Z., \& Yue, X. (2019). Constrained three-way approximations of fuzzy sets: From the perspective of minimal distance. Information Sciences, 502, 247-267. https://doi.org/10.1016/j.ins.2019.06.004

[20] Ban, A. I., \& Coroianu, L. (2012). Nearest interval, triangular and trapezoidal approximation of a fuzzy number preserving ambiguity. International Journal of Approximate Reasoning, 53(5), 805-836.

[21] Peker, S., \& Nasibov, E. (2019). Tip II genelleştirilmiş çan şekilli bulanık sayısının Tip II parametrik yamuk bulanık sayı yakınsanması. Süleyman Demirel Üniversitesi Fen Bilimleri Enstitüsü Dergisi, 23(1), 163-169. https://doi.org/10.19113/sdufenbed.466901

[22] Huang, S., Zhao, G., \& Chen, M. (2018). A fast analytical approximation type-reduction method for a class of spiked concave type-2 fuzzy sets. International Journal of Approximate Reasoning, 103, 212-226. https://doi.org/10.1016/j.ijar.2018.10.002

[23] Wang, C. Y., \& Wan, L. (2018). Type-2 fuzzy implications and fuzzy-valued approximation reasoning. International Journal of Approximate Reasoning, 102, 108-122. https://doi.org/10.1016/j.jjar.2018.08.004

[24] Dutta, P., \& Limboo, B. (2017). Bell-shaped fuzzy soft sets and their application in medical diagnosis. Fuzzy Information and Engineering, 9(1), 67-91. https://doi.org/10.1016/j.fiae.2017.03.004 